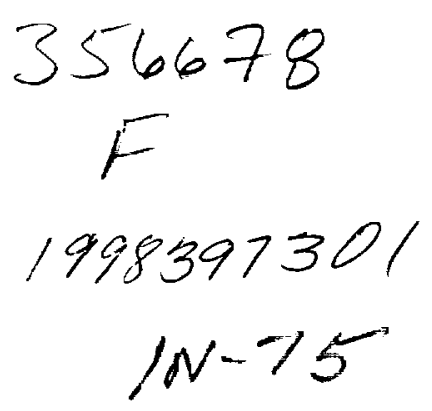

\title{
Convection of Plasmaspheric Plasma into the Outer Magnetosphere and Boundary Layer Region: Initial Results

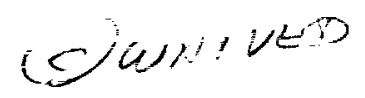

Daniel M. Ober, ${ }^{1,2}$ J. L. Horwitz

CSPAAR, University of Alabama in Huntsville, Huntsville, Alabama

D. L. Gallagher

Space Science Laboratory, NASA Marshall Space Flight Center, Huntsville, Alabama

We present initial results on the modeling of the circulation of plasmasphericorigin plasma into the outer magnetosphere and low-latitude boundary layer (LLBL), using a dynamic global core plasma model (DGCPM). The DGCPM includes the influences of spatially and temporally varying convection and refilling processes to calculate the equatorial core plasma density distribution throughout the magnetosphere. We have developed an initial description of the electric and magnetic field structures in the outer magnetosphere region. The purpose of this paper is to examine both the losses of plasmaspheric-origin plasma into the magnetopause boundary layer and the convection of this plasma that remains trapped on closed magnetic field lines. For the I.LBL electric and magnetic structures we have adopted here, the plasmaspheric plasma reaching the outer magnetosphere is diverted anti-sunward primarily along the dusk flank. These plasmas reach $\mathrm{X}=-15 \mathrm{R}_{\mathrm{E}}$ in the LL.BL approximately 3.2 hours after the initial enhancement of convection and continues to populate the LLBL for 12 hours as the convection electric field diminishes.

\section{INTRODUCTION}

a. - Convection of cold plasma through the inner magnetosphere can be broadly divided into two different flow regimes by the separatrix between open and closed drift paths. Flux tubes on closed drift paths convect

\footnotetext{
${ }^{1}$ Current address is Space Science Laboratory, NASA Marshall Space Flight Center, Huntsville, Alabama.

${ }^{2}$ NAS/NRC Research Associate.
}

Geospace Mass and Energy Flow: Results From the International Solar-Terrestrial Physics Program Geophysical Monograph 104 Copyright 1998 by the American Geophysical Union eastward with the earth filling to high $\left(\sim 10^{3} \mathrm{~cm}^{-3}\right)$ densities [e.g., Nishida, 1966]. Flux lubes on open drift paths convect sunward from the tail region, filling to comparatively lower densities $\left(-1-10 \mathrm{~cm}^{-3}\right)$, before being lost to the outer magnetosphere. During large-scale convection enhancements, the separatrix between open and closed drift paths penetrates closer to the earth. Plasmaspheric flux tubes on previously closed drift paths may now be located outside the separatrix and convect sunward on open drift paths into the outer magnetosphere creating a plasmaspheric tail [Chen and Wolf, 1972] or possibly a 'detached' plasma region [Chappell, 1974]. Freeman et al. [1977] were probably the firsts to suggest that detached plasmaspheric clouds [e.g., Chappell, 1974] could circulate from the dusk bulge region during large- 
scale storm-time convection gust into the outer magnetosphere, magnetosheath, and low-latitude boundary layer (LLBL) regions. Their scenario further proposed that such plasmas would be energized, and convect into the tail region, so that the plasmasphere could thus be a viable source of energetic magnetospheric plasmas.

There have been several observations for which spacecraft crossed the magnetopause and enhanced density regions were observed next to and just inside the initial magnetopause crossing and in between successive multiple crossings for a single pass [Chappell, 1974]. Adjacent observations of plasmaspheric plasmas and magnetopause crossings have also been reported from geosynchronous orbit during times of high magnetospheric activity [Elphic et al., 1996]. The presence of $\mathrm{He}^{+}$in the magnetospheric boundary layers can perhaps be used to identify the loss of plasmaspheric plasma out of the magnetosphere, since $\mathrm{He}^{+}$ is a significant component of plasmaspheric plasma [Horwitz et al., 1984]. Observations from ISEE 1 have shown $\mathrm{He}^{+}$densities near the subsolar magnetopause of 0.2 $\mathrm{cm}^{-3}, \mathrm{He}^{+}$densities inside the magnetopause of $0.8 \mathrm{~cm}^{-3}$, and significant $\mathrm{He}^{+}$concentrations for two cases in the magnetosheath layer outside the magnetopause [Peterson et al., 1982]. Observations from AMPTE showed cold $\mathrm{He}^{+}$ ions at densities in the range $0.1-0.8 \mathrm{~cm}^{-3}$ convecting into the subsolar low latitude boundary layer where it was evidently heated and accelerated to energies of a few $\mathrm{keV}$ [Fuselier et al., 1989]. Observations on PROGNOZ-7 have shown cases where the cold plasma component was frequently found to dominate the local magnetospheric plasma density in the dayside boundary layer indicated by a high percentage of $\mathrm{He}^{+}$ions [Lundin et al,, 1985]. This cold plasma component may have the largest influence on the flow of momentum across the boundary layer [Lundin et al., 1985; Lundin et al., 1984].

Here we seek to explore quantitatively the circulation of plasmaspheric plasma into the outer magnetosphere and boundary layer regions using a dynamic global core plasma model (DGCPM) [Ober et al., 1997; Ober et al., 1995]. We present the results for a sequence in which the large-scale convection increases following a magnetically quiet period. First, we will discuss the formulation of the model magnetic and electric field structures in the outer magnetosphere regions used in the DGCPM, and then the implications of our model results will be discussed.

\section{MODEL DESCRIPTION}

The electric field structure used here for the DGCPM derives from a time-dependent ionospheric two-cell electric potential model [Sojka et al., 1986] which is mapped along assumed equipotential magnetic field lines given by the Tsyganenko-89 model $(\mathrm{Kp}=0)$ [Tsyganenko, 1989]. These magnetospheric electric and magnetic field structures are used to calculate the EXB drift of approximately 140,000 convecting cold plasma flux tubes. The plasma density is assumed uniform along a flux tube and the content of a tube is controlled by ionospheric refilling and tube volume variations during convection. The total ion content of a magnetic flux tube evolves in time as

$$
\frac{D_{\perp} N}{D t}=\frac{F_{N}+F_{S}}{B_{i}}
$$

where $D / D t$ is the convective derivative in the moving frame of the flux tube, $\mathrm{N}$ is the total ion content per unit magnetic flux, $F_{N}$ and $F_{S}$ are the ionospheric fluxes in or out of the tube at the northern and southern ionospheres, and $B_{i}$ is the magnetic field in the ionosphere at the foot point of the flux tube [Rasmussen, 1993; Chen and Wolf, 1972]. The net flux of particles into the flux tube on the dayside is

$$
F_{D}=\frac{n_{\text {sat }}-n}{n_{\text {sat }}} F_{\max }
$$

where $F_{D}\left(=F_{N}+F_{S}\right)$ is the dayside ionospheric flux, $n_{\text {sat }}$ is the saturation density of plasma in the flux tube, $n$ is the density of plasma in the flux tube, and $F_{\max }\left(=2.9 \cdot 10^{8}\right.$ particles $/ \mathrm{cm}^{2} / \mathrm{sec}$ ) is the limiting flux of particles from the ionospheres [Rasmussen, 1992; Chen and Wolf, 1972]. The saturation density is approximated from the empirical model of Carpenter and Anderson [1992]. The nightside flux is approximated as exponential drainage of the flux tube content into the nightside ionosphere.

Observations of the LLBL from IMP 6 indicate that the bulk flow always has an anti-sunward component, that the LLBL is at times on closed field lines, and that the thickness appears to increase with increasing longitudinal distance from the subsolar point [Eastman et al., 1979]. Flow speeds are largest close to the magnetopause [Sckopke et al., 1981]. We can achieve these characteristics for our model LLBL by allowing closed magnetic field lines in the outer magnetospheric flanks and subsolar region to map into the ionosphere around the polar cap boundary and flow reversal region. Magnetic field lines in the LLBL are expected to have significant field aligned potential drops. Here the electric potential is mapped assuming equipotential field lines. The effects of field aligned potential drops on the structure of the electric field in the LLBL will be considered in future work. Figure 1 shows the model electric potential pattern in the ionosphere for a total polar cap potential drop of $72 \mathrm{kV}$. The shaded region in Figure 1 shows approximately the area in our model ionosphere that maps to the outer magnetosphere and LLBL regions. The LLBL flow velocities in our model are on the order of $0-300$ 
$\mathrm{km} / \mathrm{sec}$ while the magnetospheric flow velocities in the outer magnetosphere tend to be in the range of $0-60 \mathrm{~km} / \mathrm{sec}$ [e.g., Eastman et al, 1979]. Figure 2 shows the electric potential pattern in the equatorial magnetosphere mapped from the ionosphere for a total polar cap potential drop of 72 $\mathrm{kV}$. The dashed line shows the inner boundary of the LLBL. Within the model LLBL, the cold plasma flow is away from the subsolar point and anti-sunward along the flanks. The total potential drop across our model LLBL shown in Figure 2, at MLT $=15$, is $26 \mathrm{kV}$ which is slightly larger than what has been observed [e.g., Hapgood and Lockwood, 1993; Mozer, 1984]. The width of the model LLBL increases with increasing anti-sunward distance.

For the preliminary investigation here, the ionospheric electric potential changes in time but not the magnetic field structure. The location of the inner boundary of the LLBL is determined by the mapping of the ionospheric electric potential pattern and changes as the electric potential evolves in time. The location of the magnetopause is determined by the transition from closed to open magnetic field lines in the magnetic field model and does not change in time. Therefore, the width of the ILBL may become unrealistically large $\left(2.6 \mathrm{R}_{\mathrm{E}}\right.$ at $\left.15 \mathrm{MLT}\right)$ at times in the simulation but we anticipate that the physics of the model still remains useful to beginning to understand the flow of plasmaspheric plasma into the outer magnetosphere and boundary layer regions.

For the simulation presented here we consider a case in which the total polar cap potential drop increases suddenly and then decays. Figure 3 shows the total polar cap

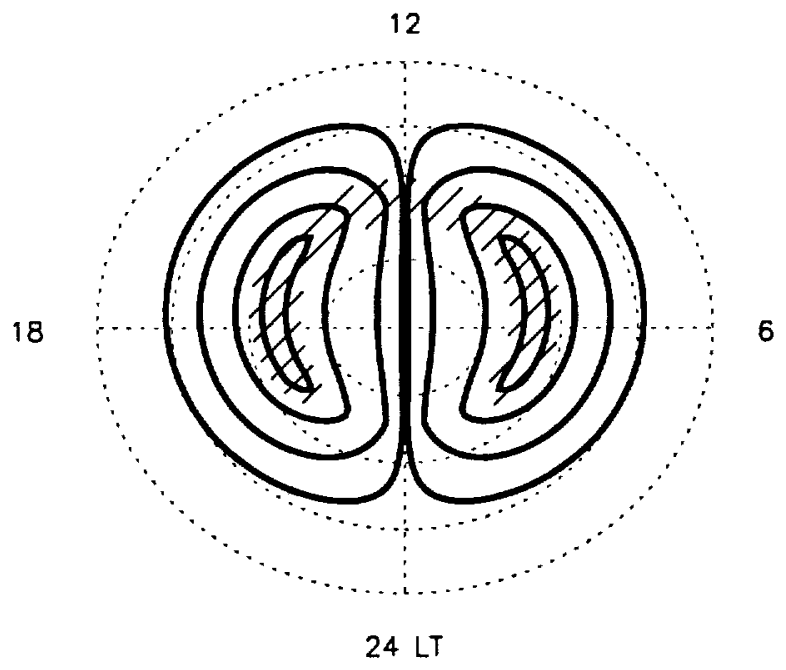

Figure 1. Electric potential pattern in the ionosphere for a total polar cap potential of $72 \mathrm{kV}$. The shaded region shows the approximate area that maps to the outer magnetosphere and LLBL regions.

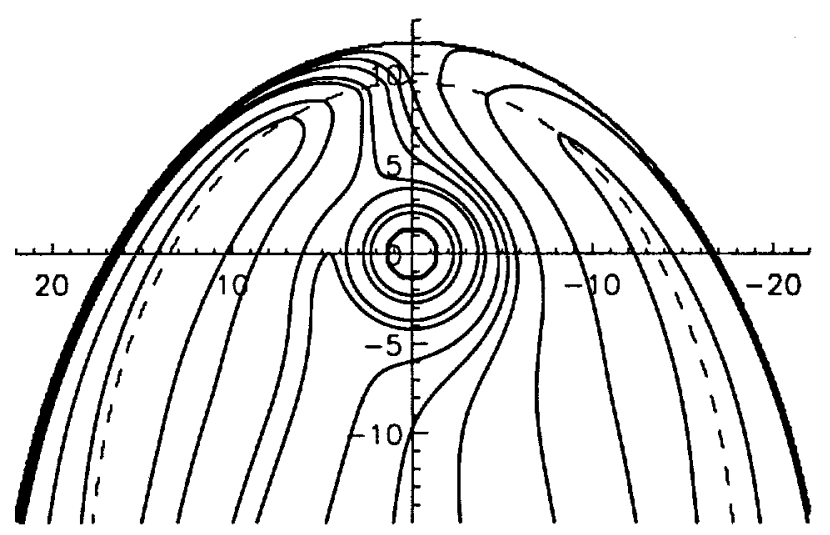

Figure 2. Electric potential pattern in the equatorial magnetosphere for a total polar cap potential of $72 \mathrm{kV}$. The dashed line shows the inner boundary of the LLBL.

potential drop, the total potential drop across the LLBL and the width of the LLBL at MLT=15, all as a function of time. The polar cap potential shown in Figure 3 rises suddenly during the first 6 hours of the simulation.

\section{RESULTS}

The simulation shown here will illustrate the convection of bulge region plasmaspheric plasma into the dayside magnetopause boundary layer. Initially, the simulation is ran with a constant potential pattern until a self-consistent steady state density distribution is established that is consistent with observations of a quiet time plasmasphere.

Plate 1 shows the initial (0 hours) density distribution in the magnetosphere prior to the sudden rise of the total polar cap potential. At 3 hours in the simulation plasmaspheric flux tubes convecting sunward into the outer magnetosphere reach the dayside magnetopause and are diverted towards the dusk flank of the magnetosphere. At 3.2 hours into the simulation, anti-sunward streaming flux tubes of plasmaspheric origin in the LLBL reach $x=-15 R_{E}$. Shown here is the continued transport of plasmaspheric origin plasma in the LL.BL at 5 hours into the simulation. At 15 hours the sunward transport of plasmaspheric flux tubes has diminished and at 21 hours the plasmasphere has returned to a near quiet time configuration except that the plasmapause has shifted earthward.

In our model simulation the cold plasma densities in the LLBL were in the range of $1-10 \mathrm{~cm}^{-3}$. Using a $\mathrm{He}^{+} / \mathrm{H}^{+}$ratio of 0.2 that is typical of the plasmasphere [Horwitz et al., 1984], our model would predict a $\mathrm{He}^{+}$density in the LLBL of $0.2-2 \mathrm{~cm}^{-3}$. This is in the range of observed $\mathrm{He}^{+}$densities in the LLBL [e.g. Peterson et al., 1982; Fuselier et al., 1989]. 


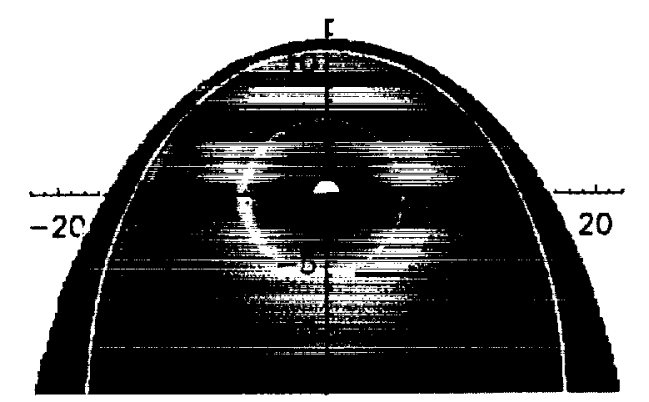

0 Hrs.

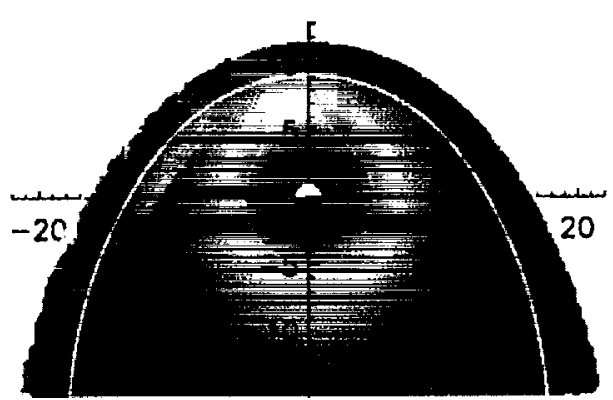

$5 \mathrm{Hrs}$.

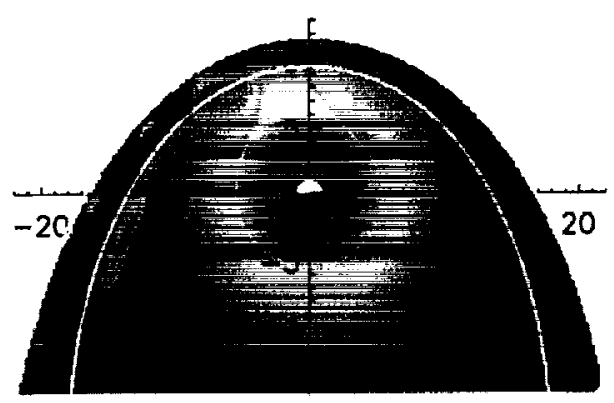

3 Hrs.

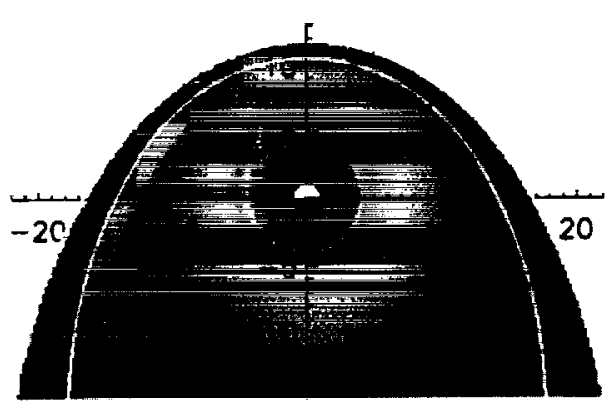

21 Hrs.

Plate 1. Modeled cold plasma density distribution in the equatorial magnetosphere at $0,3,5$, and 21 hours during the simulation. The white line marks the location of the inner edge of the low-latitude boundary layer. The axis are geocentric in units of $\mathrm{R}_{\mathrm{E}}$. Color bar shows density color scale used.

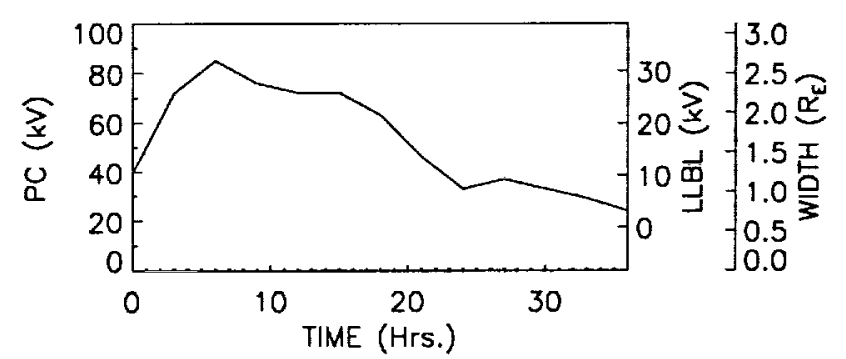

Figure 3. Plotted are the total polar cap potential drop, the total potential drop across the LLBL and the width of the LIBL, both at MLT $=15$, used in the model simulation.

\section{CONCLUSION}

In our model simulation it was observed that plasmaspheric flux tubes convect sunward from the dusk bulge region during a large-scale convection enhancement and are diverted along the dusk flank in the LLBL when reaching the outer magnetosphere. Plasmaspheric flux tubes convecting in the IL.BL region require about 20 minutes to reach $X=-15 R_{E}$. Transport of plasmaspheric origin flux tubes into the dusk flank LLBL continues for about 12 hours until the outer plasmasphere has become depleted. The modeled $\mathrm{He}^{+}$densities in the LLBL are around $0.2-2 \mathrm{~cm}^{-3}$ that is in the range of observed values of total densities appropriate to $\mathrm{He}^{+}$in the LLBL. In our simulation we have only considered the convection of plasma that remains on closed field lines. Flux tubes in the outer plasmasphere may also convect out to the magnetopause, reconnect with the IMF, and convect over the polar cap [Elphic et al., 1997; Freeman et al., 1977].

Acknowledgments. This research was supported by NASA grant NGT-51299 and NSF grant ATM-9301024 to the University of Alabama in Huntsville.

\section{REFERENCES}

Carpenter, D. L., and R. R. Anderson, An ISEE/whistler model of equatorial electron density in the magnetosphere, J. Geophy. Res., 97, 1157, 1992.

Chappell, C. R., Detached plasma regions in the magnetosphere, J. Geophy. Res., 79, 1861, 1974.

Chen, A. J., and R. A. Wolf, Effects on the plasmasphere of a timevarying convection electric field, Planet. Space Sci., 20, 483, 1972.

Elphic, R. C., M. F. Thomsen, and J. E. Borovsky, The fate of the outer plasmasphere, Geophys. Res. Iett., 24, 365, 1997. 
Elphic, R. C., L. A. Weiss, M. F. Thomsen, D. J. McComas, and M. B. Moldwin, Evolution of plasmaspheric ions at geosynchronous orbit during times of high geomagnetic activity, Geophys. Res. Lett., 23, 2189, 1996.

Eastman, T. E., and E. W. Hones, Jr., Characteristics of the magnetospheric boundary layer and magnetopause layer as observed by Imp 6, J. Geophys. Res., 84, 2019, 1979.

Freeman, J. W., H. K. Hills, T. W. Hill, and P. H. Reiff, Heavy ion circulation in the earth's magnetosphere, Geophys. Res. Lett., 4, 195, 1977.

Fuselier, S. A., W. K. Peterson, D. M. Klumpar, and E. G. Shelley, Entry and acceleration of $\mathrm{He}+$ in the low latitude boundary layer, Geophys. Res. Lett., 16, 751, 1989.

Hapgood, M. and M. Lockwood, On the voltage and distance across the low latitude boundary layer, Geophys. Res. Lett., 20, 145, 1993.

Horwitz, J. L., R. H. Comfort, and C. R. Chappell, Thermal ion composition measurements of the formation of the new outer plasmasphere and double plasmapause during the storm recovery phase, Geophys. Res. Lett., 11, 704, 1984.

Lundin, R., E. M. Dubinin, Solar wind energy transfer regions inside the dayside magnetopause: Accelerated heavy ions as tracers for MHD-processes in the dayside boundary layer, Planet. Space Sci., 33, 891, 1985.

Lundin, R., E. M. Dubinin, Solar wind energy transfer regions inside the dayside magnetopause-1. Evidence for magnetospheric plasma penetration, Planet. Space Sci., 32, 745, 1984.

Mozer, F. S. Electric field evidence on the viscous interaction at the magnetopause, Geophys. Res. Let., 11, 135, 1984.

Ober, D. M., J. L. Horwitz and D. L. Gallagher, Formation of density troughs embedded in the outer plasmasphere by subauroral ion drifts (SAID), J. Geophys. Res., 14595, 1997.
Ober, D. M., J. L. Horwitz and D. L. Gallagher, Global plasmasphere evolution during a sub-auroral ion drift (SAID) event, in Physics of Space Plasmas (1995). Number 14, T. Chang and J. R. Jasperse, eds. (MIT Center for Theoretical Geo/Cosmo Plasma Physics, Cambridge, MA, 1996), 691.

Nishida, A., Formation of plasmapause, or magnetospheric knee, by the combined action of magnetospheric convection and plasma escape from the tail, J. Geophys. Res., 71, 5669, 1966.

Peterson, W. K., E. G. Shelley, G. Haerendel, and G. Paschmann, Energetic ion composition in the subsolar magnetopause and boundary layer, J. Geophy. Res., 87, 2139, 1982.

Rasmussen, C. E., S. M. Guitar, and S. G. Thomas, A twodimensional model of the plasmasphere: Refilling time constants, Planet. Space Sci., 41, 35, 1993.

Rasmussen, C. E., The plasmasphere, In Physics of space plasmas, numberl 2 in SPI Conference proceedings and reprint series, page 279 , Scientific publishers, 1992.

Sckopke, N., G. Paschmann, G. Haerendel, B. U. O. Sonnerup, S J. Bame, T. G. Forbes, E. W. Hones, Jr., and C. T. Russell, Structure of the low-latitude boundary layer, J. Geophy. Res., $86,2099,1981$

Sojka, J. J., C. E. Rasmussen, and R. W. Schunk, An interplanetary magnetic field dependent model of the ionospheric convection electric field, J. Geophy. Res., 91, 11281, 1986.

Tsyganenko, N. A., A magnetospheric magnetic field model with a warped tail current sheet, Planet. Space Sci., 37, 5, 1989.

D. L. Gallagher, and D. M. Ober, ES83, Space Science Laboratory, NASA Marshall Space Flight Center, Huntsville, Al 35812.

J. I. Horwitz, CSPAAR, The University of Alabama in Huntsville, Huntsville, Al 35899. 
(1)
$=-$

$\vdots$

||| 